\title{
Symmetric bowtie decompositions of the complete graph
}

\author{
Simona Bonvicini \\ Dipartimento di Scienze e Metodi dell'Ingegneria \\ Via Amendola 2, Pad. Morselli, 42100 Reggio Emilia, Italy \\ simona.bonvicini@unimore.it \\ Beatrice Ruini \\ Dipartimento di Matematica Pura ed Applicata \\ Via Campi 213/b, 41125 Modena, Italy \\ beatrice.ruini@unimore.it
}

Submitted: Nov 23, 2009; Accepted: Jul 7, 2010; Published: Jul 20, 2010

Mathematics Subject Classification: 05C25, 05B07, 20B25

\begin{abstract}
Given a bowtie decomposition of the complete graph $K_{v}$ admitting an automorphism group $G$ acting transitively on the vertices of the graph, we give necessary conditions involving the rank of the group and the cycle types of the permutations in $G$. These conditions yield non-existence results for instance when $G$ is the dihedral group of order $2 v$, with $v \equiv 1,9(\bmod 12)$, or a group acting transitively on the vertices of $K_{9}$ and $K_{21}$. Furthermore, we have non-existence for $K_{13}$ when the group $G$ is different from the cyclic group of order 13 or for $K_{25}$ when the group $G$ is not an abelian group of order 25. Bowtie decompositions admitting an automorphism group whose action on vertices is sharply transitive, primitive or 1-rotational, respectively, are also studied. It is shown that if the action of $G$ on the vertices of $K_{v}$ is sharply transitive, then the existence of a $G$-invariant bowtie decomposition is excluded when $v \equiv 9(\bmod 12)$ and is equivalent to the existence of a $G$-invariant Steiner triple system of order $v$. We are always able to exclude existence if the action of $G$ on the vertices of $K_{v}$ is assumed to be 1-rotational. If, instead, $G$ is assumed to act primitively then existence can be excluded when $v$ is a prime power satisfying some additional arithmetic constraint.
\end{abstract}

\section{Introduction}

A bowtie is a simple graph with 5 vertices and 6 edges consisting of a pair of edge-disjoint cycles (called triples) sharing one vertex. A bowtie decomposition of the complete graph 
$K_{v}=(V, E)$ is a partition $\mathcal{B}_{v}$ of the edge-set $E$ into bowties. Such a decomposition $\mathcal{B}_{v}$ exists if and only if $v \equiv 1,9(\bmod 12)$, see [2]. Such values of $v$, with $v>1$, will be called admissible values.

For non-admissible values, some authors considered the problem of selecting a collection of edge-disjoint bowties in $K_{v}$ of maximum cardinality (the so called maximum packing problem of $K_{v}$ with bowties, see [3]). Bowtie decompositions with additional properties have also been considered. For instance, 2-perfect bowtie decompositions have been studied in [4], [1].

In the present paper we are interested in bowtie decompositions with some symmetry properties. These are namely properties involving in the first place the existence of some non-trivial automorphism group.

An automorphism group $G$ of a bowtie decomposition $\mathcal{B}_{v}$ is a subgroup of the symmetric group $\operatorname{Sym}(v)$ leaving the decomposition invariant: we shall express that for short by saying that $\mathcal{B}_{v}$ is $G$-invariant. A group of automorphisms of a decomposition $\mathcal{B}_{v}$ acts on four different sets: the vertices, the edges, the triples and the bowties. In this paper we will focus our attention on the action of the group on vertices. More specifically, we study bowtie decompositions admitting an automorphism group $G$ whose action on vertices is transitive, sharply transitive, primitive or 1-rotational, respectively. Here 1-rotational means that $G$ fixes one vertex of $K_{v}$ and acts sharply transitively on the remaining ones. We adopt the terminology used in [5].

By a result in [11], for every admissible value of $v$ a Steiner triple system of order $v$, briefly $\operatorname{STS}(v)$, can be decomposed into bowties. In fact, from [11] we know that the block intersection graph of an $\operatorname{STS}(v)$ is Hamiltonian. Since $v$ is an admissible value, the block intersection graph has an even number of vertices: it is therefore possible to pair the triples of an $\operatorname{STS}(v)$ so as to decompose the $\operatorname{STS}(v)$ into bowties, or equivalently, to obtain a bowtie decomposition of $K_{v}$. For this reason one is tempted to believe that, in order to find bowtie decompositions with a prescribed automorphism group, it suffices to pair the triples of an $\operatorname{STS}(v)$ which is invariant with respect to the same group. Unfortunately, the existence of an $\operatorname{STS}(v)$ which is invariant under a group $G$ does not guarantee that we can pair its triples and obtain a $G$-invariant bowtie decomposition. In fact, the $\operatorname{STS}(v)$ might have one $G$-orbit $O$ of triples in which $|O|$ is odd and different from the lengths of all other $G$-orbits of triples. Hence, if we pair a triple in $O$ with a triple in a $G$-orbit different from $O$ we obtain a bowtie whose $G$-orbit contains at least two distinct bowties sharing a triple. The same thing happens if we pair two distinct triples in $O$. In other words, every conceivable pairing of the triples of the $\operatorname{STS}(v)$ yields a bowtie decomposition which is never $G$-invariant.

The converse is true, that is a $G$-invariant bowtie decomposition $\mathcal{B}_{v}$ gives rise to an $\operatorname{STS}(v)$ admitting $G$ as an automorphism group.

In Section 2 transitive bowtie decompositions are studied. A bowtie decomposition $\mathcal{B}_{v}$ is transitive if it admits an automorphism group $G$ acting transitively on vertices. If we need to mention the group $G$ explicitly, we shall then say that $\mathcal{B}_{v}$ is transitive with respect to $G$. The bowties of a transitive bowtie decomposition satisfy certain properties, see Lemma 1 and 2 . These properties yield a necessary condition on the rank $r$ of $G$ : 
$r \equiv 1(\bmod 3)$ or $r \equiv 1(\bmod 12)$, according to whether $|G|$ is even or odd. As a trivial consequence, there is no bowtie decomposition which is invariant with respect to a group acting 2-transitively on the vertex-set. We also exclude the existence of transitive bowtie decompositions of $K_{13}$ with respect to a group $G$ of order $>13$.

We shall show that a transitive bowtie decomposition of $K_{v}$ exists only if the number $s$ of self-paired orbitals of $G$ is less than $(r-1) / 3$. In this way we can exclude the existence of a transitive bowtie decomposition $\mathcal{B}_{v}$ which is invariant with respect to the dihedral group of order $2 v$.

Many papers deal with STS $(v)$ 's with a single automorphism of prescribed type. More specifically, given a permutation $\pi \in \operatorname{Sym}(v)$, with a prescribed cycle type, an $\operatorname{STS}(v)$ admitting $\pi$ as an automorphism is constructed. See for instance [17], [8], [16], [6] and [13].

In Section 3, given a decomposition $\mathcal{B}_{v}$ which is transitive with respect to a group $G$, we study the cycle types of the permutations in $G$. The necessary conditions we give exclude the existence of transitive bowtie decompositions of $K_{9}$ and $K_{21}$. We can also say that there is no transitive bowtie decomposition of $K_{25}$ with respect to a group $G$ of order $>25$.

In Section 4 sharply transitive bowtie decompositions are studied. A decomposition $\mathcal{B}_{v}$ is sharply transitive if it admits an automorphism group $G$ acting sharply transitively on vertices. If we need to mention the group $G$ explicitly, we shall then say that $\mathcal{B}_{v}$ is sharply transitive with respect to $G$. We adopt the same terminology for STS $(v)$ 's.

An easy calculation shows that the existence of a sharply transitive decomposition $\mathcal{B}_{v}$ with $v \equiv 9(\bmod 12)$ can be excluded. For the other admissible values of $v$, the existence of a sharply transitive decomposition $\mathcal{B}_{v}$ turns out to be equivalent to the existence of a sharply transitive $\operatorname{STS}(v)$ (see Proposition 14). In this way, a large class of examples for sharply transitive bowtie decompositions of $K_{v}$, with $v \equiv 1(\bmod 12)$, can be obtained from the abelian $\operatorname{STS}(v)$ 's constructed in [19].

Even though transitive STS $(v)$ 's are widely studied, little is known when the automorphism groups under consideration are non-abelian. In the framework of transitive $\operatorname{STS}(v)$ 's ad hoc treatments for particular values of $v$ have appeared. For instance, in [15] the transitive STS(21) are determined. The authors showed that there are 10 nonisomorphic transitive $\operatorname{STS}(21)$. Later, Tonchev, [20], proved that when $v=25$ the number of non-isomorphic transitive $\operatorname{STS}(v)$ 's is 15.

Finding transitive bowtie decompositions is equivalent to finding transitive $\operatorname{STS}(v)$ 's with additional properties. The determination of the spectrum for transitive bowtie decompositions is still an open question: our necessary conditions of Section 3 yield nonexistence results for all admissible values of $v \leqslant 30$.

Section 5 is devoted to primitive bowtie decompositions of $K_{v}$. A decomposition $\mathcal{B}_{v}$ is primitive if it admits an automorphism group acting primitively on vertices. The groups we consider are of affine type and have order $p^{n} q$, where $p$ is a prime such that $v=p^{n} \equiv 1$ $(\bmod 12)$ and $q$ is a $p$-primitive divisor of $p^{n}-1$. When $(v-1) / q \not \equiv 0(\bmod 12)$ we have a non-existence result. When $(v-1) / q \equiv 0(\bmod 12)$ we can exhibit some examples of bowtie decompositions which are invariant with respect to a primitive group $G$ of the type 
described above.

Finally, we consider 1-rotational bowtie decompositions of $K_{v}$. A decomposition $\mathcal{B}_{v}$ is 1 -rotational if it admits an automorphism group whose action on the vertices of $K_{v}$ is 1-rotational. We prove that 1-rotational bowtie decompositions of $K_{v}$ exist for no admissible value of $v$.

\section{Transitive bowtie decompositions: a rank condi- tion}

Let $K_{v}=(V, E)$ be the complete graph on $v$ vertices. In this section, we shall denote by $v$ an admissible value and by $G$ a subgroup of the symmetric group $\operatorname{Sym}(v)$ acting transitively on the vertex-set of $K_{v}$.

The group $G$ acts on $V \times V$ via $(x, y)^{g}=\left(x^{g}, y^{g}\right)$ and an orbit of $G$ on $V \times V$ is an orbital of $G$. The number of orbitals of $G$ is the rank of $G$, which we shall denote by $r$.

Each orbital $\Delta$ of $G$ defines its paired orbital $\Delta^{*}=\{(\beta, \alpha):(\alpha, \beta) \in \Delta\}$. An orbital $\Delta$ is self-paired if $\Delta=\Delta^{*},[9]$. Observe that, since $G$ acts transitively on $V$, the set $\Delta_{1}=\{(\alpha, \alpha): \alpha \in V\}$ is an orbital, which is obviously self-paired and is called the diagonal orbital.

By the previous remarks, we can write $r=2 t+s+1$, where $2 t$ is the number of non-self-paired orbitals, $s$ is the number of non-diagonal self-paired orbitals and 1 refers to the diagonal orbital $\Delta_{1}$.

For any given bowtie $B$ of $K_{v}$ we consider the 5 -tuple $\left(x, y_{1}, z_{1}, y_{2}, z_{2}\right)$ of its vertices and use it to identify $B$ uniquely by agreeing that $x$ is the unique 4 -valent vertex of $B$ (i.e. vertex of degree 4 ) called the centre of $B$, while $T_{1}=\left(x, y_{1}, z_{1}\right)$ and $T_{2}=\left(x, y_{2}, z_{2}\right)$ are the triples of $B$.

We shall use the exponential notation for edge-orbits and bowtie-orbits under the action of $G$ and its subgroups.

We shall denote by $S$ the stabilizer of $B$ in $G$. Every element of $S$ fixes the centre $x$ of $B$, since $x$ is the unique 4 -valent vertex of $B$.

It is easy to see that two distinct edges, say $\left[u_{1}, w_{1}\right],\left[u_{2}, w_{2}\right]$, belong to the same $G-$ orbit if and only if the ordered pair $\left(u_{1}, w_{1}\right)$ belongs to the same orbital as either $\left(u_{2}, w_{2}\right)$ or $\left(w_{2}, u_{2}\right)$.

An edge $[u, w] \in E$ is said to be short if the pair $(u, w)$ belongs to a self-paired orbital of $G$.

An edge $[u, w] \in E$ is said to be long if the pair $(u, w)$ belongs to a non-self-paired orbital of $G$.

Note that short edges exist if and only if the group $G$ has even order. In fact, if $G$ has even order then there is at least one permutation $g \in G$ of order 2 ; if $(u w)$ is a transposition appearing in the representation of $g$ as a product of disjoint cycles then the orbital containing the ordered pair $(u, w)$ is self-paired and the edge $[u, w]$ is short. It is easy to see that also the converse is true. 
Lemma 1. Let $\mathcal{B}_{v}$ be a transitive decomposition with respect to $G$. Let $B=\left(x, y_{1}, z_{1}, y_{2}, z_{2}\right)$ be a bowtie of $\mathcal{B}_{v}$. The following properties hold:

(a) the edges of $B$ which are incident with the centre $x$ are all long;

(b) the edge-orbit $\left[y_{i}, z_{i}\right]^{G}$, with $i=1,2$, does not contain edges which are incident with the centre $x$ of $B$.

Proof. We prove property $(a)$. Suppose that $\left[x, y_{i}\right]$, with $i \in\{1,2\}$, is short. Then there exists $g \in G$ such that $x^{g}=y_{i}$ and $y_{i}^{g}=x$. Whence $g \in S$, a contradiction, since we have remarked that every element of $S$ fixes $x$. Hence property $(a)$ is true. Property $(b)$ is handled similarly.

As a consequence of the previous lemma, the following statement holds.

Lemma 2. Let $\mathcal{B}_{v}$ be a transitive decomposition with respect to $G$. Let $B=\left(x, y_{1}, z_{1}, y_{2}, z_{2}\right)$ be a bowtie of $\mathcal{B}_{v}$. Then $B$ satisfies one of the following properties:

(1) the edges $\left[y_{i}, z_{i}\right], i=1,2$, are short and have the same $G$-orbit; the edges $\left[x, y_{i}\right]$ and $\left[x, z_{i}\right], i=1,2$, are long and have the same $G$-orbit;

(2) the edges $\left[y_{i}, z_{i}\right], i=1,2$, are short and have distinct $G$-orbits; the edges $\left[x, y_{1}\right]$, $\left[x, z_{1}\right],\left[x, y_{2}\right],\left[x, z_{2}\right]$ are long, belong to 2 distinct $G$-orbits and $\left[x, y_{i}\right]^{G}=\left[x, z_{i}\right]^{G}$, for $i=1,2$;

(3) the edges $\left[y_{i}, z_{i}\right], i=1,2$, are long and have the same $G$-orbit; $\left[x, y_{1}\right],\left[x, y_{2}\right],\left[x, z_{1}\right]$, $\left[x, z_{2}\right]$ are long, belong to 2 distinct $G$-orbits and $\left[x, y_{i}\right]^{G} \neq\left[x, z_{i}\right]^{G}$, for $i=1,2$;

(4) the edges $\left[y_{i}, z_{i}\right], i=1,2$ are long and have distinct $G$-orbits; the edges $\left[x, y_{1}\right],\left[x, z_{1}\right]$, $\left[x, y_{2}\right],\left[x, z_{2}\right]$ are long and belong to 4 distinct $G$-orbits;

(5) the edge $\left[y_{1}, z_{1}\right]$ is short; the edges $\left[x, y_{1}\right],\left[x, z_{1}\right]$ are long and have the same $G$-orbit; the edges $\left[x, y_{2}\right],\left[x, z_{2}\right],\left[y_{2}, z_{2}\right]$ are long and belong to 3 distinct $G$-orbits.

We shall say that a bowtie $B$ of $\mathcal{B}_{v}$ is of type $(i), i \in\{1, \ldots, 5\}$, with respect to $G$ if $B$ satisfies property $(i)$ of Lemma 2. When the group $G$ is clear from the context, we simply say that a bowtie is of type $(i)$.

Proposition 1. Let $\mathcal{B}_{v}$ be a transitive decomposition with respect to $G$. Let $r$ be the rank of $G$. If $|G|$ is even, then $r \equiv 1(\bmod 3)$, otherwise $r \equiv 1(\bmod 12)$.

Proof. Let $\mathcal{R}=\left\{B_{1}, \ldots, B_{\mu}\right\}$ be a complete system of distinct representatives for the $G$-orbits of $\mathcal{B}_{v}$.

For $i=1, \ldots, 5$, we denote by $a_{i}$ the number of elements of $\mathcal{R}$ of type $(i)$. If $|G|$ is odd then there are no short edges, hence $a_{1}=a_{2}=a_{5}=0$, but also $a_{3}=0$. In fact, suppose $a_{3} \neq 0$ that is $\mathcal{B}_{v}$ possesses a bowtie $B=\left(x, y_{1}, z_{1}, y_{2}, z_{2}\right)$ of type $(3)$, then there is a permutation $g \in G$ such that $\left[y_{1}, z_{1}\right]^{g}=\left[y_{2}, z_{2}\right]$; whence $B^{g}=B$, as $\mathcal{B}_{v}$ is $G$-invariant, that is $\left[y_{2}, z_{2}\right]^{g}=\left[y_{1}, z_{1}\right]$; it follows that $g$ has even order, a contradiction. 
Since $\mathcal{B}_{v}$ is a partition of the edge-set of $K_{v}$, for every non-diagonal orbital $\Delta$ of $G$ there exists a unique bowtie $B_{i} \in \mathcal{R}$ containing the edge $[x, y]$ such that $(x, y)^{G}=\Delta$ or $(y, x)^{G}=\Delta$. Moreover, $\cup_{j=1}^{\mu} \cup_{[x, y] \in B_{j}}\left((x, y)^{G} \cup(y, x)^{G}\right)=(V \times V) \backslash \Delta_{1}$. Hence we can write the number $s$ of self-paired orbitals of $G$ as $s=a_{1}+2 a_{2}+a_{5}$, since the bowties of type (1), (2) and (5) are the only ones possessing short edges. For the same reason, we have $t=a_{1}+2 a_{2}+3 a_{3}+6 a_{4}+4 a_{5}$. Whence $t-s=3\left(a_{3}+2 a_{4}+a_{5}\right)$, that is $t-s=3 q$, with $q \geqslant 0$, if $|G|$ is even, otherwise $t=6 a_{4}$. Hence $r=2 t+s+1=3(2 q+s)+1$, if $|G|$ is even, otherwise $r=12 a_{4}+1$.

Corollary 1. Let $G$ be a group of odd order and rank $r<13$. There is no transitive bowtie decomposition $\mathcal{B}_{v}$ with respect to $G$.

Proposition 2. For $s>(r-1) / 3$ there is no transitive bowtie decomposition of $K_{v}$.

Proof. From the proof of Proposition 1, we can see that if there exists a bowtie decomposition which is invariant with respect to a transitive group $G$, then $s \leqslant t$. Hence $r-1=2 t+s \geqslant 3 s$, that is $s \leqslant(r-1) / 3$.

The next statement furnishes an example in which Proposition 2 applies.

Proposition 3. Let $v$ be an admissible value. There is no decomposition $\mathcal{B}_{v}$ which is transitive with respect to the dihedral group of order $2 v$.

Proof. We identify the vertex-set of $K_{v}$ with $\mathbb{Z}_{v}=\{0,1, \ldots, v-1\}$. We label consecutively by $0,1, \ldots, v-1$, clockwise, the vertices of the regular $v$-gon in the euclidian plane. The edges of $K_{v}$ are given by the chords and the sides of the polygon.

Let $G$ denote the dihedral group of order $2 v$ in its standard permutation representation, that is $G=\langle\rho, \theta\rangle$, where $\rho$ and $\theta$ are the permutations on $\mathbb{Z}_{v}$ defined by $\rho: x \mapsto x+1$ and $\theta: x \mapsto-x+1$, respectively. The cyclic group $\langle\rho\rangle$ consists of the permutations on $\mathbb{Z}_{v}$ of the form $\rho^{i}: x \mapsto x+i$, for every $i=0, \ldots, v-1$. The coset $G-\langle\rho\rangle$ consists of the permutations on $\mathbb{Z}_{v}$ of the form $x \mapsto-x+i+1$. Each element of $G-\langle\rho\rangle$ is a reflection about the axis passing through the centre of the polygon and the vertex $j$, for every $j=0, \ldots, v-1$.

It is easy to see that the set $\left\{[0, i+1]: i=0, \ldots, \frac{v-3}{2}\right\}$ is a complete system of distinct representatives for the $G$-orbits of edges. Moreover, each edge $[0, i+1]$ is short, since $[0, i+1]^{\theta \rho^{i}}=[i+1,0]$. Hence $\left\{(0, i+1): i=0, \ldots, \frac{v-3}{2}\right\}$ is a complete system of distinct representatives for the non-diagonal orbitals of $G$. Whence $s=(v-1) / 2=r-1$. The assertion follows from Proposition 2.

Using the AllTransitiveGroups library of GAP, [10], we can see that the groups $G$ acting transitively on 13 vertices, other than the cyclic group of order 13, have rank $r=2,3,4,5$ or 7 , respectively. The existence of a transitive bowtie decomposition is excluded by Proposition 1 if $G$ has rank $r=2,3$ or 5 and by Propositions 2 or 3 if $G$ has rank $r=4$ or 7 , respectively. It is known that for $v=13$ there exists a sharply transitive $\operatorname{STS}(v)$ under the cyclic group of order 13, [18]. We shall see in Proposition 14 that the existence of a sharply transitive bowtie decomposition is equivalent to the existence of a 
sharply transitive $\operatorname{STS}(v)$. Hence there exists a sharply transitive bowtie decomposition under the cyclic group of order 13. Therefore, the following statement holds.

Proposition 4. Let $G$ be a transitive permutation group on 13 vertices. If $|G|>13$ then no $G$-invariant bowtie decomposition of $K_{13}$ exists.

\section{The cycle type of an automorphism of a $\mathcal{B}_{v}$}

In this section $v$ will be an admissible value and $G \leqslant \operatorname{Sym}(v)$ will be a group acting transitively on the vertex-set of $K_{v}$.

A permutation $g \in G$ is said to be of type $[g]=\left[f, p_{2}, \ldots, p_{v-1}, p_{v}\right]$ if $g$ fixes $f \geqslant 0$ vertices and $p_{k}$ is the number of $k$-cycles in the representation of $g$ as a product of disjoint cycles.

Given a permutation $g \in G$, when we will speak of a " $k$-cycle of $g$ " we will always mean a $k$-cycle in the representation of $g$ as a product of disjoint cycles.

It is easy to see that if $\mathcal{B}_{v}$ is a bowtie decomposition of $K_{v}$ which is invariant with respect to $G$, then $\mathcal{B}_{v}$ gives rise to a Steiner triple system $\mathcal{S}$ of order $v$ which is invariant with respect to $G$ : it suffices to split every bowtie of $\mathcal{B}_{v}$ into its two triples.

One can see that if an automorphism $g$ of $\mathcal{S}$ fixes $f \geqslant 1$ vertices, then $f \equiv 1,3(\bmod 6)$. In fact, if a triple $T$ of $\mathcal{S}$ contains two vertices which are fixed by $g$, then all vertices of $T$ are fixed by $g$, since $\mathcal{S}$ is invariant under $g$. Hence the triples of $\mathcal{S}$ all of whose vertices are fixed by $g$ give an $S T S(f)$. Hence $f \equiv 1,3(\bmod 6)$, [7], and the following result holds.

Lemma 3. Let $g \in G$ be an automorphism of $\mathcal{B}_{v}$ fixing $f>0$ vertices. Then $f \equiv 1,3$ $(\bmod 6)$.

Proof. It follows from the fact that an automorphism of $\mathcal{B}_{v}$ is an automorphism of $\mathcal{S}$.

We give necessary conditions for the existence of a transitive bowtie decomposition.

Proposition 5. Let $g \in G$ be an automorphism of $\mathcal{B}_{v}$ of type $[g]=\left[f, p_{2}, \ldots, p_{v}\right]$, with $f>1$ and $p_{2}=0$. Then $f \equiv 1,9(\bmod 12)$.

Proof. Let $F$ be the set of vertices of $K_{v}$ which are fixed by $g$. We have $|F|=f \geqslant 2$.

Let $z_{1}, z_{2}$ be distinct elements of $F$ and let $B$ denote the unique bowtie of $\mathcal{B}_{v}$ containing the edge $\left[z_{1}, z_{2}\right]$. Then $B^{g}=B$, since $B$ and $B^{g}$ share the edge $\left[z_{1}, z_{2}\right]$ and $\mathcal{B}_{v}$ is invariant under $g$. Whence all vertices of $B$ are fixed by $g$, since $p_{2}=0$. We have thus proved that the bowties of $\mathcal{B}_{v}$ containing at least one edge with endpoints in $F$ have all edges with both endpoints in $F$. In other words, a bowtie of $\mathcal{B}_{v}$ has either no edge with endpoints in $F$ or all edges with endpoints in $F$. Hence $\mathcal{B}_{v}$ induces a bowtie decomposition $\mathcal{B}_{f}$ of $K_{F}$. Hence $f \equiv 1,9(\bmod 12),[2]$.

Proposition 6. Let $g \in G$ be an automorphism of $\mathcal{B}_{v}$ of type $[g]=\left[f, p_{2}, \ldots, p_{v}\right]$ with $p_{2} \neq 0$. Then $f \geqslant 1$ and $p_{2}+f(f-1) / 6$ is even. 
Proof. Let $\mathcal{S}$ be the $\operatorname{STS}(v)$ arising from $\mathcal{B}_{v}$. Since $p_{2} \neq 0$, there exist at least two distinct vertices of $K_{v}$, say $x, y$, such that $x^{g}=y$ and $y^{g}=x$. We have $[x, y]^{g}=[x, y]$ and, if $B$ is the unique bowtie of $\mathcal{B}_{v}$ containing $[x, y]$, we also have $B^{g}=B$. The centre of $B$ is the unique 4 -valent vertex of $B$ and is thus fixed by $g$, yielding $f \geqslant 1$.

We denote by $F$ the set of vertices of $K_{v}$ which are fixed by $g$ and by $\left(x_{1} y_{1}\right), \ldots$, $\left(x_{p_{2}} y_{p_{2}}\right)$ the 2 -cycles (transpositions) of $g$.

For every $i=1, \ldots, p_{2}$, there exists a triple $T_{i} \in \mathcal{S}$ containing the edge $\left[x_{i}, y_{i}\right]$, since $\mathcal{S}$ is a partition of the edge-set of $K_{v}$. We set $T_{i}=\left(x_{i}, y_{i}, z_{i}\right)$. We have that $z_{i} \in F$, since $\left[x_{i}, y_{i}\right]^{g}=\left[x_{i}, y_{i}\right]$ and $\mathcal{S}$ is invariant with respect to $g$. It might happen that $z_{i}=z_{j}$ for $i, j \in\left\{1, \ldots, p_{2}\right\}$, with $i \neq j$, that is $T_{i}$ and $T_{j}$ share one vertex. We set $\mathcal{T}=\left\{T_{i}: i=\right.$ $\left.1, \ldots, p_{2}\right\}$.

As remarked at the beginning of this section, the triples of $\mathcal{S}$ with all vertices in $F$ give an $\operatorname{STS}(f)$, say $\mathcal{S}^{\prime}$.

We show that if one of the triples of a bowtie in $\mathcal{B}_{v}$ lies in $\mathcal{T} \cup \mathcal{S}^{\prime}$, then so does the other triple.

Let $T_{1} \in \mathcal{T} \cup \mathcal{S}^{\prime}$ and let $B$ be the bowtie of $\mathcal{B}_{v}$ containing $T_{1}$. We denote by $T_{2}$ the other triple of $B$. If $T_{1} \in \mathcal{S}^{\prime}$, then the centre of $B$ lies in $F$. The same occurs if $T_{1} \in \mathcal{T}$, since $g$ fixes $B$. Hence at least one vertex of $T_{2}$ lies in $F$. Since $T_{1}^{g}=T_{1}$, as $T_{1} \in \mathcal{T} \cup \mathcal{S}^{\prime}$, we have $B^{g}=B$, that is $T_{2}^{g}=T_{2}$. Whence the remaining two vertices of $T_{2}$ are either in $F$ or appear together in a 2 -cycle of $g$, that is $T_{2} \in \mathcal{T} \cup \mathcal{S}^{\prime}$.

We have thus proved that a bowtie of $\mathcal{B}_{v}$ contains 0 or 2 triples of $\mathcal{T} \cup \mathcal{S}^{\prime}$. Whence $\left|\mathcal{T} \cup \mathcal{S}^{\prime}\right|=p_{2}+f(f-1) / 6$ is even.

Proposition 7. Let $\mathcal{B}_{v}$ be a transitive decomposition with respect to $G$. Let $g \in G$ be a permutation of even order $o(g)$ and type $[g]=\left[f, p_{2}, \ldots, p_{v}\right]$. Then $f+\sum_{k \in Q} k p_{k}>1$, where $Q=\left\{k: 2 \leqslant k \leqslant v, k \mid \frac{o(g)}{2}\right\}$ and it is empty if $o(g)=2$.

Proof. Let $\mathcal{S}$ be the $\operatorname{STS}(v)$ which arises from $\mathcal{B}_{v}$. We set $\alpha=o(g) / 2$.

Let $k \in\{2, \ldots, v\}$ be such that $p_{k} \neq 0$. We can write $o(g)=k q$, where $q$ is a positive integer. We note that if $k$ is odd, then $q$ is even, as $o(g)$ is even, whence $\alpha=k \frac{q}{2}$, that is $k \mid \alpha$. Hence, if $k \notin Q$ then $k$ is even.

We write $v=f+\sum_{k=2}^{v} k p_{k}=f+\sum_{k \in Q} k p_{k}+\sum_{k \notin Q} k p_{k}$ and show firstly that $f+\sum_{k \in Q} k p_{k}>0$, secondly that $f+\sum_{k \in Q} k p_{k}>1$.

Suppose $f+\sum_{k \in Q} k p_{k}=0$. Then $v=\sum_{k \notin Q} k p_{k}$. That yields a contradiction, since $v$ is odd and $\sum_{k \notin Q} k p_{k}$ is even, as $k \notin Q$. Hence $f+\sum_{k \in Q} k p_{k} \geqslant 1$.

We show that $f+\sum_{k \in Q} k p_{k}>1$. Suppose $f+\sum_{k \in Q} k p_{k}=1$. Let $h=g^{\alpha}$. We have that $h$ is an involution of $G$ fixing exactly $f+\sum_{k \in Q} k p_{k}$ vertices of $K_{v}$. In fact, the vertices which are fixed by $h$ are given by the vertices which are fixed by $g$ together with the vertices lying in the $k$-cycles of $g$ with $k \mid \alpha$, that is $k \in Q$. Since we are supposing $f+\sum_{k \in Q} k p_{k}=1$, the involution $h$ fixes only one vertex of $K_{v}$, that is $h$ is the product of $m=(v-1) / 2$ disjoint 2-cycles.

We denote by $z$ the unique vertex of $K_{v}$ which is fixed by $h$ and write $h$ as the disjoint product $h=\left(x_{1} y_{1}\right)\left(x_{2} y_{2}\right) \ldots\left(x_{m} y_{m}\right)$. 
For every $i=1, \ldots, m$, the edge $\left[x_{i}, y_{i}\right]$ is short, since it is left invariant by $h$. Moreover, for every $i=1, \ldots, m$, there is exactly one triple $T_{i} \in \mathcal{S}$ containing the edge $\left[x_{i}, y_{i}\right]$. Each $T_{i}$ is fixed by $h$, since $\mathcal{S}$ is invariant under $h$. Whence $T_{i}=\left(x_{i}, y_{i}, z\right)$, for every $i=1, \ldots, m$.

Since $G$ is transitive on $V$, there exists $g_{1} \in G$ such that $z^{g_{1}}=x_{1}$. Then $z^{g_{1} h g_{1}^{-1}}=$ $x_{1}^{h g_{1}^{-1}}=y_{1}^{g_{1}^{-1}} \neq z$, since $x_{1} \neq y_{1}$. Hence $z^{g_{1} h g_{1}^{-1}}=x_{j}$ or $z^{g_{1} h g_{1}^{-1}}=y_{j}$, with $j \in\{1, \ldots, m\}$. It follows that the edge $\left[z, z^{g_{1} h g_{1}^{-1}}\right]$ is left invariant by $g_{1} h g_{1}^{-1}$, since $g_{1} h g_{1}^{-1}$ is an involution mapping $z$ to $z^{g_{1} h g_{1}^{-1}}$. In other words, the edge $\left[z, z^{g_{1} h g_{1}^{-1}}\right]$ is short. Then the triple $T_{j}=$ $\left(x_{j}, y_{j}, z\right)$ contains two short edges: $\left[x_{j}, y_{j}\right]$ and $\left[z, z^{g_{1} h g_{1}^{-1}}\right]$. That yields a contradiction, since by property $(a)$ in Lemma 1 a triple of a bowtie can contain at most one short edge. Hence $f+\sum_{k \in Q} k p_{k}>1$.

Corollary 2. Let $\mathcal{B}_{v}$ be a transitive bowtie decomposition which is invariant with respect to $G$. The number of fixed vertices of each involution in $G$ is $>1$ and $\equiv 1,3(\bmod 6)$.

Proof. An involution $g$ of $G$ is a permutation of type $[g]=\left[f, p_{2}, 0, \ldots, 0\right]$, with $p_{2} \neq 0$. The set $Q=\left\{k: 2 \leqslant k \leqslant v, k \mid \frac{o(g)}{2}\right\}$ is empty, hence $f=f+\sum_{k \in Q} k p_{k}>1$, since Proposition 7 holds. The statement follows from Lemma 3.

Proposition 8. Let $\mathcal{B}_{v}$ be a transitive decomposition with respect to $G$. Let $g \in G$ be a permutation of even order o(g) and type $[g]=\left[f, p_{2}, \ldots, p_{v}\right]$. Let $k \in\{3, \ldots, v\}$ be such that $k \equiv 2(\bmod 4), k \nmid \frac{o(g)}{2}$ and $p_{k} \neq 0$. Then

$$
\sum_{\left(h, k_{2}\right) \in \mathcal{D}_{k}} \operatorname{gcd}\left(h, k_{2}\right) p_{h} p_{k_{2}}+\frac{1}{4} p_{\frac{k}{2}}\left(k p_{\frac{k}{2}}-2\right)+f p_{\frac{k}{2}}-3 p_{k} \equiv 0,3 \quad(\bmod 6)
$$

where $\mathcal{D}_{k}=\left\{\left(k_{1}, k_{2}\right): k_{1}<k_{2}, k_{i} \neq k / 2\right.$, for $\left.i=1,2, \frac{k_{1} k_{2}}{\operatorname{gcd}\left(k_{1}, k_{2}\right)}=\frac{k}{2}\right\} \cup\left\{\left(\frac{k}{2}, k_{2}\right): k_{2} \mid \frac{k}{2}, k_{2} \neq\right.$ $\left.1, \frac{k}{2}\right\}$.

Proof. We set $\alpha=o(g) / 2$ and $h=g^{\alpha}$. Observe that $h$ is an involution of $G$. Let $k \in\{3, \ldots, v\}$ be such that $p_{k} \neq 0$. As remarked in the proof of the previous proposition, if $k \nmid \alpha$ then $k$ is even and every $k$-cycle of $g$ gives rise to $k / 2$ disjoint 2 -cycles of $h$. Every 2 -cycle $(x y)$ of $h$ yields the short edge $[x, y]$. For the sake of brevity, we shall say that an edge $[x, y]$ is a $k$-short edge if $(x y)$ is a 2 -cycle of $h$ which arises from a $k$-cycle of $g$. Note that a $k$-short edge $[x, y]$ has $\langle g\rangle$-orbit of length $k / 2$, since $x, y$ lie in a $k$-cycle of $g$ and $[x, y]^{h}=[x, y]$. An edge $[x, y]$ which is not $k$-short and has $\langle g\rangle$-orbit of length $k / 2$ will be called $k$-long. We denote by $\gamma$ the number of distinct $\langle g\rangle$-orbits of $k$-long edges. Firstly, we prove that $\gamma-3 p_{k} \equiv 0,3(\bmod 6)$; secondly, we compute the number $\gamma$.

Let $\mathcal{A}_{k}$ be the subset of $\mathcal{B}_{v}$ consisting of all bowties containing at least one edge with $\langle g\rangle$-orbit of length $k / 2$. Let $\mathcal{R}$ be a complete system of distinct representatives for the $\langle g\rangle$-orbits of $\mathcal{A}_{k}$. We denote by $a_{i}$ the number of elements of $\mathcal{R}$ which are of type $(i)$, $i \in\{1,2, \ldots, 5\}$, with respect to $\langle g\rangle$. An easy calculation shows that $a_{1}=0$ since $k \equiv 2$ $(\bmod 4)$.

By the very definition of $\mathcal{A}_{k}$, we have that $\mathcal{A}_{k}$ covers all edges with $\langle g\rangle$-orbit of length $k / 2$, hence the following relations hold: $2 a_{2}+a_{5}=p_{k}$ and $3 a_{3}+6 a_{4}+3 a_{5}=\gamma$. 
The former equality arises from the fact that the number of distinct $\langle g\rangle$-orbits of $k_{-}^{-}$ short edges is $p_{k}$, that a bowtie of type (5) has a non-empty intersection with only one $\langle g\rangle$-orbit of $k$-short edges, while a bowtie of type (2) has a non-empty intersection with exactly two distinct $\langle g\rangle$-orbits of $k$-short edges.

The latter equality arises from the fact that every bowtie of type (3) and (5) has a non-empty intersection with exactly three distinct $\langle g\rangle$-orbits of $k$-long edges, while every bowtie of type (4) has a non-empty intersection with six distinct $\langle g\rangle$-orbits of $k$-long edges.

Substituting $a_{5}=p_{k}-2 a_{2}$ in $3 a_{3}+6 a_{4}+3 a_{5}=\gamma$, we find that $\gamma-3 p_{k} \equiv 0,3(\bmod 6)$.

We determine the number $\gamma$, that is the number of distinct $\langle g\rangle$-orbits of $k$-long edges. Let $[x, y]$ be a $k$-long edge. For the vertices $x, y$ we distinguish three cases.

Case 1: $x$ lies in a $k_{1}$-cycle of $g, y$ lies in a $k_{2}$-cycle of $g$ with $k_{1}, k_{2} \neq k / 2$ and such that $\frac{k_{1} k_{2}}{\operatorname{gcd}\left(k_{1}, k_{2}\right)}=k / 2$.

We denote by $\gamma_{1}$ the number of distinct $\langle g\rangle$-orbits of $k$-long edges whose vertices $x, y$ satisfy the property of Case 1.

We prove that $\gamma_{1}=\sum_{\left(k_{1}, k_{2}\right) \in J} \operatorname{gcd}\left(k_{1}, k_{2}\right) p_{k_{1}} p_{k_{2}}$, where $J=\left\{\left(k_{1}, k_{2}\right): k_{1}<k_{2}, k_{i} \neq\right.$ $k / 2$, for $\left.i=1,2, \frac{k_{1} k_{2}}{\operatorname{gcd}\left(k_{1}, k_{2}\right)}=\frac{k}{2}\right\}$.

Let $[x, y]$ be an edge such that $x$ is one of the vertices of the $k_{1}$-cycle $\left(x_{1} \ldots x_{k_{1}}\right)$ and $y$ is one of the vertices of the $k_{2}-$ cycle $\left(y_{1} \ldots y_{k_{2}}\right)$. The edge $[x, y]$ is of type $\left[x_{a}, y_{b}\right]$ with $a \in\left\{1, \ldots, k_{1}\right\}$ and $b \in\left\{1, \ldots, k_{2}\right\}$. Every $\left[x_{a}, y_{b}\right]^{\langle g\rangle}$ contains at least one edge which is incident with $x_{1}$. Hence to determine the representatives of the distinct $\langle g\rangle$-orbits of edges $\left[x_{a}, y_{b}\right]$, with $a \in\left\{1, \ldots, k_{1}\right\}$ and $b \in\left\{1, \ldots, k_{2}\right\}$, we can consider the edges $\left[x_{1}, y_{1}\right]$, $\left[x_{1}, y_{2}\right], \ldots,\left[x_{1}, y_{k_{2}}\right]$.

We note that $\left[x_{1}, y_{b}\right]^{\langle g\rangle}$, with $b \in\left\{1, \ldots, k_{2}\right\}$, contains $\frac{k}{2} / k_{1}=\frac{k_{2}}{\operatorname{gcd}\left(k_{1}, k_{2}\right)}$ edges which are incident with $x_{1}$, since $x_{1}$ lies in a $k_{1}$-cycle of $g$ and $\left[x_{a}, y_{b}\right]^{\langle g\rangle}$ has length $k / 2$. In other words, $\left[x_{1}, y_{b}\right]^{\langle g\rangle}$ contains the edges $\left[x_{1}, y_{b+j k_{1}}\right]$, for every $j=0, \ldots, \frac{k_{2}}{\operatorname{gcd}\left(k_{1}, k_{2}\right)}-1$. These edges are pairwise distinct. In fact, assume $\left[x_{1}, y_{b+j k_{1}}\right]=\left[x_{1}, y_{b+j^{\prime} k_{1}}\right]$, with $j, j^{\prime} \in$ $\left\{0, \ldots, \frac{k_{2}}{\operatorname{gcd}\left(k_{1}, k_{2}\right)}-1\right\}, j \neq j^{\prime}$. Without loss of generality we can set $j>j^{\prime}$. We have $y_{b+j k_{1}}=y_{b+j^{\prime} k_{1}}$, that is $b+j k_{1}=b+j^{\prime} k_{1}$, whence $\left(j-j^{\prime}\right) k_{1}=q k_{2}$, for some positive integer $q$. Since $k_{1} \neq k / 2$, as $\left(k_{1}, k_{2}\right) \in J$, we have that $\left(j-j^{\prime}\right) k_{1}=q k_{2}$ if and only if $j-j^{\prime} \geqslant \frac{k_{2}}{\operatorname{gcd}\left(k_{1}, k_{2}\right)}$. That yields a contradiction, since $j-j^{\prime} \leqslant \frac{k_{2}}{\operatorname{gcd}\left(k_{1}, k_{2}\right)}-1$. We have thus proved that every $\left[x_{1}, y_{b}\right]^{\langle g\rangle}$ contains $\frac{k_{2}}{\operatorname{gcd}\left(k_{1}, k_{2}\right)}$ distinct edges which are incident with $x_{1}$. Therefore, among $\left[x_{1}, y_{1}\right],\left[x_{1}, y_{2}\right], \ldots,\left[x_{1}, y_{k_{2}}\right]$ we have $k_{2} / \frac{k_{2}}{\operatorname{gcd}\left(k_{1}, k_{2}\right)}=\operatorname{gcd}\left(k_{1}, k_{2}\right)$ distinct representatives of $\langle g\rangle$-orbits of edges. In other words, every pair of cycles of $g$ of length $k_{1}, k_{2}$, with $\left(k_{1}, k_{2}\right) \in J$, gives $\operatorname{gcd}\left(k_{1}, k_{2}\right)$ distinct representatives. Since the number of cycles of $g$ of length $k_{1}, k_{2}$ is $p_{k_{1}}, p_{k_{2}}$, respectively, and $\left(k_{1}, k_{2}\right) \in J$ we find $\sum_{\left(k_{1}, k_{2}\right) \in J} \operatorname{gcd}\left(k_{1}, k_{2}\right) p_{k_{1}} p_{k_{2}}$ distinct representatives of $\langle g\rangle$-orbits of $k$-long edges. Note that in $J$ the condition $k_{1}<k_{2}$ assures that in $\sum_{\left(k_{1}, k_{2}\right) \in J} \operatorname{gcd}\left(k_{1}, k_{2}\right) p_{k_{1}} p_{k_{2}}$ the edges are counted one time.

Case 2: $x$ lie in a $\frac{k}{2}$-cycle of $g, y$ lies in a $k_{2}$-cycle of $g$ with $k_{2} \neq k / 2$ and $k_{2} \mid \frac{k}{2}$.

We denote by $\gamma_{2}$ the number of distinct $\langle g\rangle$-orbits of $k$-long edges whose vertices $x, y$ satisfy the property of Case 2. This case can be treated as Case 1: it suffices to replace 
$k_{1}$ by $\frac{k}{2}$ and $p_{k_{2}}$ by $f$ if $y$ is fixed by $g$. We find that $\gamma_{2}=f p_{\frac{k}{2}}+\sum_{k_{2} \in J^{\prime}} k_{2} p_{\frac{k}{2}} p_{k_{2}}$, where $J^{\prime}=\left\{k_{2}: k_{2} \mid \frac{k}{2}, k_{2} \neq 1, \frac{k}{2}\right\}$.

Case 3: $x, y$ lie in $\frac{k}{2}$-cycles of $g$ (distinct or not).

We denote by $\gamma_{3}$ the number of distinct $\langle g\rangle$-orbits of $k$-long edges whose vertices $x, y$ satisfy the property of Case 3 . We prove that $\gamma_{3}=\frac{k}{2} p_{\frac{k}{2}}\left(p_{\frac{k}{2}}-1\right) / 2+p_{\frac{k}{2}}(k-2) / 4$.

Let $[x, y]$ be an edge such that $x, y$ lie in distinct $\frac{k}{2}$-cycles of $g$, say $\left(x_{1} \ldots x_{\frac{k}{2}}\right)$, $\left(y_{1} \ldots y_{\frac{k}{2}}\right)$, respectively. Hence $[x, y]$ is of type $\left[x_{a}, y_{b}\right]$, with $a, b \in\left\{1, \ldots, \frac{k}{2}\right\}$. Every $\left[x_{a}, y_{b}\right]^{\langle g\rangle}$ contains exactly one edge which is incident with $x_{1}$, since $x_{1}$ lies in a $\frac{k}{2}$-cycle of $g$ and $\left[x_{a}, y_{b}\right]^{\langle g\rangle}$ has length $k / 2$. Hence we can take $\left[x_{1}, y_{1}\right],\left[x_{1}, y_{2}\right], \ldots,\left[x_{1}, y_{\frac{k}{2}}\right]$ as the representatives of the distinct $\langle g\rangle$-orbits of edges $\left[x_{a}, y_{b}\right]$, with $a, b \in\left\{1, \ldots, \frac{k}{2}\right\}$. In other words, for every pair of distinct $\frac{k}{2}$-cycles of $g$ we find $k / 2$ distinct representatives. Since the number of pairs of distinct $\frac{k}{2}$-cycles of $g$ is $p_{\frac{k}{2}}\left(p_{\frac{k}{2}}-1\right) / 2$, we find $\frac{k}{2} p_{\frac{k}{2}}\left(p_{\frac{k}{2}}-1\right) / 2$ distinct representatives of $\langle g\rangle$-orbits of $k$-long edges.

Assume $x, y$ to be in the same $\frac{k}{2}$-cycle of $g$, say $\left(x_{1} \ldots x_{\frac{k}{2}}\right)$. Then $[x, y]$ is of type $\left[x_{a}, x_{b}\right]$, with $a, b \in\left\{1, \ldots, \frac{k}{2}\right\}$ and $a \neq b$. Every $\left[x_{a}, x_{b}\right]^{\langle g\rangle}$ contains at least one edge which is incident with $x_{1}$. Hence to determine the representatives of the distinct $\langle g\rangle$-orbits of edges $\left[x_{a}, x_{b}\right]$, with $a, b \in\left\{1, \ldots, \frac{k}{2}\right\}$ and $a \neq b$, we can consider the edges $\left[x_{1}, x_{2}\right]$, $\left[x_{1}, x_{3}\right], \ldots,\left[x_{1}, x_{\frac{k}{2}}\right]$. Note that for every $b=2, \ldots, \frac{k}{2}$, the edge $\left[x_{1}, x_{b}\right]$ is $k$-long, since $k \equiv 2(\bmod 4)$. Moreover, $x_{b}^{g^{\frac{k}{2}-b+1}}=x_{b+\frac{k}{2}-b+1}=x_{1}, x_{1}^{g^{\frac{k}{2}-b+1}}=x_{1+\frac{k}{2}-b+1}=x_{\frac{k}{2}-b+2}$ and $x_{\frac{k}{2}-b+2} \neq x_{b}$, as $k \equiv 2(\bmod 4)$. Hence $\left[x_{1}, x_{\frac{k}{2}-b+2}\right] \in\left[x_{1}, x_{b}\right]^{\langle g\rangle}$ and $\left[x_{1}, x_{b}\right],\left[x_{1}, x_{\frac{k}{2}-b+2}\right]$ are the only edges which are incident with $x_{1}$ which are contained in $\left[x_{1}, x_{b}\right]^{\langle g\rangle}$. Therefore, among $\left[x_{1}, x_{2}\right],\left[x_{1}, x_{3}\right], \ldots,\left[x_{1}, x_{\frac{k}{2}}\right]$ we find $\left(\frac{k}{2}-1\right) / 2=\frac{k-2}{4}$ distinct representatives. In other words, for every $\frac{k}{2}$-cycle of $g$ the number of distinct representatives for the $\langle g\rangle$ orbits of edges $\left[x_{a}, x_{b}\right]$, with $a, b \in\left\{1, \ldots, \frac{k}{2}\right\}, a \neq b$, is $\frac{k-2}{4}$. Since the number of distinct $\frac{k}{2}$-cycles is $p_{\frac{k}{2}}$, we have $p_{\frac{k}{2}}(k-2) / 4$ representatives of $\langle g\rangle$-orbits of $k$-long edges.

We have $\gamma=\gamma_{1}+\gamma_{2}+\gamma_{3}$, that is

$$
\gamma=\sum_{\left(k_{1}, k_{2}\right) \in J} \operatorname{gcd}\left(k_{1}, k_{2}\right) p_{k_{1}} p_{k_{2}}+f p_{\frac{k}{2}}+\sum_{k_{2} \in J^{\prime}} k_{2} p_{\frac{k}{2}} p_{k_{2}}+\frac{k}{2} p_{\frac{k}{2}}\left(p_{\frac{k}{2}}-1\right) / 2+p_{\frac{k}{2}} \frac{(k-2)}{4}
$$

whence

$$
\gamma=\sum_{\left(h, k_{2}\right) \in \mathcal{D}_{k}} \operatorname{gcd}\left(h, k_{2}\right) p_{h} p_{k_{2}}+\frac{1}{4} p_{\frac{k}{2}}\left(k p_{\frac{k}{2}}-2\right)+f p_{\frac{k}{2}}
$$

Proposition 9. Let $\mathcal{B}_{v}$ be a transitive decomposition with respect to $G$. Let $g \in G$ be a permutation of odd order $o(g)$ and type $[g]=\left[f, p_{2}, \ldots, p_{v}\right]$. Let $k \in\{3, \ldots, v\}$ be such that $p_{k} \neq 0$. Then 


$$
\sum_{\left(h, k_{2}\right) \in \mathcal{D}_{k}} \operatorname{gcd}\left(h, k_{2}\right) p_{h} p_{k_{2}}+\frac{1}{2} p_{k}\left(k p_{k}-1\right)+f p_{k} \equiv 0,3 \quad(\bmod 6)
$$

where $\mathcal{D}_{k}=\left\{\left(k_{1}, k_{2}\right): k_{1}<k_{2}, k_{i} \neq k\right.$, for $\left.i=1,2, \frac{k_{1} k_{2}}{\operatorname{gcd}\left(k_{1}, k_{2}\right)}=k\right\} \cup\left\{\left(k, k_{2}\right): k_{2} \mid k, k_{2} \neq\right.$ $1, k\}$.

Proof. The proof is similar to the proof of the previous proposition, with the exception that no $k$-short edge is defined, as $g$ has odd order, and that a $k$-long edge is an edge with $\langle g\rangle$-orbit of length $k$. Hence the set $\mathcal{A}_{k}$ can contain only elements of type (3) or (4) with respect to $\langle g\rangle$.

Below we give a result concerning Steiner triple systems. We shall use it in the proof of Proposition 10.

Lemma 4. Let $\mathcal{S}$ be an $S T S(v)$ which is invariant with respect to $G$. Let $g \in G$ be of type $[g]=\left[f, p_{2}, \ldots, p_{v}\right]$ with $f \geqslant 1$. Then for every odd $k \in\{3, \ldots, v\}$ we have that $p_{k}$ is even.

Proof. Assume $k \in\{3, \ldots, v\}$ with $k$ odd and $p_{k} \neq 0$. Let $z$ be a vertex of $K_{v}$ which is fixed by $g$ and let $x$ be a vertex of $K_{v}$ lying in a $k$-cycle of $g$. Let $T \in \mathcal{S}$ be the unique triple containing the edge $[z, x]$. We set $T=(z, x, y)$. Observe that $y$ is not fixed by $g$, since $g$ does not fix $x$ and $\mathcal{S}$ is invariant with respect to $g$. We show that $y$ lies in a $k$-cycle of $g$ not containing $x$.

Let $y$ be one of the vertices in a $d$-cycle of $g$, with $d \neq k$. If $d<k$, then the triples $T$ and $T^{g^{d}}$ share the edge $[z, y]$, but $T \neq T^{g^{d}}$, since $x^{g^{d}} \neq x$, as $d<k$. That yields a contradiction, since $\mathcal{S}$ is invariant with respect to $\langle g\rangle$. The same happens if $d>k$. Hence $y$ lies in a $k$-cycle of $g$.

Let $y$ be one of the vertices in the $k$-cycle of $g$ containing $x$, that is $y=x^{g^{a}}$ for some $a \in\{1, \ldots, k-1\}$. Then $T$ and $T^{g^{a}}$ share the edge $[z, y]$, but $T \neq T^{g^{a}}$ since $k$ is odd. That yields a contradiction, since $\mathcal{S}$ is invariant with respect to $\langle g\rangle$. Hence $y$ lies in a $k$-cycle of $g$ not containing $x$.

By the property proved above, every triple of $\mathcal{S}$ containing $z$ either contains no vertex from a $k$-cycle of $g$ or it contains two such vertices from distinct $k$-cycles of $g$.

Let $\mathcal{T}_{k}$ be the subset of $\mathcal{S}$ consisting of all triples containing $z$ and two vertices of $K_{v}$ lying in (distinct) $k$-cycles of $g$.

Let $T_{1}, T_{2}$ be distinct triples of $\mathcal{T}_{k}$. We set $T_{1}=\left(z, x_{1}, y_{1}\right), T_{2}=\left(z, x_{2}, y_{2}\right)$. It is easy to see that $T_{1}, T_{2}$ have the same $\langle g\rangle$-orbit if and only if $x_{1}$ lies in the same $k$-cycle of $x_{2}$ (or $y_{2}$ ). Observe that $x_{1}$ lies in the same $k$-cycle of $x_{2}$ (or $y_{2}$ ) if and only if $y_{1}$ lies in the same $k$-cycle of $y_{2}\left(\right.$ or $\left.x_{2}\right)$, since $\mathcal{S}$ is invariant with respect to $\langle g\rangle$.

Let $\left\{T_{1}, \ldots, T_{\mu}\right\}$ be a complete system of distinct representatives for the $\langle g\rangle$-orbits of $\mathcal{T}_{k}$. By the very definition of $\mathcal{T}_{k}$ and by the remarks above, $2 \mu \leqslant p_{k}$. For every $k$-cycle $c$ of $g$ there is a unique triple in $\left\{T_{1}, \ldots, T_{\mu}\right\}$ containing exactly one vertex lying in $c$, since $\mathcal{T}_{k}$ is a partition of the edges having precisely one vertex in a $k$-cycle of $g$. Hence $p_{k} \leqslant 2 \mu$, then $p_{k}=2 \mu$. 
Proposition 10. Let $\mathcal{B}_{v}$ be a transitive decomposition with respect to $G$. Let $g \in G$ be of type $[g]=\left[f, p_{2}, \ldots, p_{v}\right]$ with $f \geqslant 1$ and $p_{3} \neq 0$. Then $p_{3} \geqslant 4$.

Proof. By Lemma 4 we have $p_{3} \geqslant 2$. Suppose $p_{3}=2$. We denote by $\left(x_{1} x_{2} x_{3}\right)$ and $\left(\begin{array}{lll}y_{1} & y_{2} & y_{3}\end{array}\right)$ the 3 -cycles of $g$.

Let $\mathcal{S}$ be the $\operatorname{STS}(v)$ arising from $\mathcal{B}_{v}$. Let $T=\left(x_{1}, x_{2}, x\right)$ be the unique triple of $\mathcal{S}$ containing the edge $\left[x_{1}, x_{2}\right]$.

Since $x_{1}$ and $x_{2}$ lie in the same 3 -cycle of $g$ and $\mathcal{S}$ is invariant with respect to $g$, we have that $x$ is not fixed by $g$.

Since the $\langle g\rangle$-orbit of the edge $\left[x_{1}, x_{2}\right]$ has length 3 and $\mathcal{S}$ is invariant with respect to $\langle g\rangle$, we have that $x$ lies in a $3-$ cycle of $g$. But $x \neq x_{3}$, since $T$ is a triple of a bowtie and by Lemma 2 such a triple can contain at most two edges in the same $G$-orbit. Hence $x=y_{i}$ for some $i \in\{1,2,3\}$. Without loss of generality, we can set $x=y_{1}$.

There exists a unique $T_{1} \in \mathcal{S}$ containing the edge $\left[y_{1}, y_{2}\right]$. We set $T_{1}=\left(y_{1}, y_{2}, y\right)$. By the previous remarks, we have $y=x_{i}$ for some $i \in\{1,2,3\}$. We have $y=x_{3}$, since $T, T_{1}$ are distinct, $T$ contains the edges $\left[x_{1}, y_{1}\right],\left[x_{2}, y_{1}\right]$ and $\mathcal{S}$ is a partition of the edge-set of $K_{v}$. Whence $T^{g}$ and $T_{1}$ are distinct triples of $\mathcal{S}$ sharing the edge $\left[x_{3}, y_{2}\right]$. That yields a contradiction, since $\mathcal{S}$ is invariant with respect to $\langle g\rangle$. Hence $p_{3}>2$. By Lemma 4 we have $p_{3} \geqslant 4$.

The AllTransitiveGroups library of GAP, [10], contains representatives for all transitive permutation groups on at most 30 vertices.

Using this library we tested the necessary conditions in Section 2 and 3 on the groups acting transitively on $v$ vertices, where $v$ is an admissible value, with $v \leqslant 30$. We remarked in Section 1 that doubly transitive bowtie decompositions do not exist, so we discarded 2 -transitive groups.

For $v=25$, the CycleStructurePerm command allows us to see that if $G$ is not an abelian group of order 25 then $G$ possesses at least one permutation $g$ satisfying one of the following properties:

1) $g$ is of type $[g]=[25-5 j, 0,0,0, j, 0, \ldots, 0]$, with $j \in\{2,3,4\}$;

2) $g$ is of type $[g]=[1,12,0, \ldots, 0]$;

3) $g$ is of type $[g]=[5,10,0, \ldots, 0]$;

4) $g$ is of type $[g]=[1,0,8,0, \ldots, 0]$;

5) $g$ is of type $[g]=\left[f, p_{2}, \ldots, p_{25}\right]$, with $f=0, p_{5}=1, p_{10}=2, p_{i}=0$ for $i=2, \ldots, 25$, $i \neq 5,10$.

If $G$ possesses one permutation satisfying property 1), then there is no transitive bowtie decomposition with respect to $G$, since $25-5 j \not \equiv 1,9(\bmod 12)$, for every $j=2, \ldots, 4$, and Proposition 5 holds. The same thing happens if $G$ possesses one permutation satisfying property 2), 3), 4) or 5), since one of Corollary 2, Proposition 9, Proposition 8 holds in each case, respectively. 
It is known that for $v=25$ there is a sharply transitive $\operatorname{STS}(25)$ with respect to $G$, when $G$ is an abelian group of order 25, [19]. By Proposition 14 an STS(25) which is invariant under a sharply transitive abelian group $G$ of order 25 yields a bowtie decomposition which is sharply transitive with respect to $G$. Hence, for $v=25$ the only examples of transitive bowtie decompositions are the sharply transitive decompositions under one of the abelian groups of order 25 (cyclic or elementary abelian).

We carried out a similar inspection for the groups $G$ acting transitively on $v$ vertices, with $v=21$ and $v=9$. In particular, for $v=9$ we use the necessary conditions of Section 3 to exclude the existence of a transitive bowtie decomposition with respect to $G$, when $G$ satisfies Proposition 1. More specifically, a group $G$ on 9 vertices has rank $r=2,3,4,5,6$ or 9. If $G$ has rank $r=2,3,5,6$ or 9 , then there is no transitive bowtie decomposition with respect $G$, since Proposition 1 holds. The groups of rank $r=4$ have even order and satisfy Proposition 1. Nevertheless, if $G$ is a group of rank $r=4$ we find at least one element $g \in G$ which is of type $[g]=[3,0,2,0, \ldots, 0]$ or of type $[g]=[6,0,1,0, \ldots, 0]$. In both cases Proposition 5 is not verified. Hence we can state the following results.

Proposition 11. Let $G$ be a transitive permutation group on 25 vertices. If $|G|>25$ then no $G$-invariant bowtie decomposition of $K_{25}$ exists.

Proposition 12. There is no transitive bowtie decomposition of $K_{9}$ and $K_{21}$.

\section{Sharply transitive bowtie decompositons}

In this section we shall denote by $v$ an admissible value and by $G$ a subgroup of $\operatorname{Sym}(v)$ acting sharply transitively on the vertices of $K_{v}$.

Observe that the stabilizer $S$ in $G$ of a bowtie $B$ consists only of the identity permutation on $V$, since every permutation in $S$ fixes the centre of $B$ and $G$ is sharply transitive on vertices.

Proposition 13. If $\mathcal{B}_{v}$ is a sharply transitive bowtie decomposition of $K_{v}$ then $v \equiv 1$ $(\bmod 12)$.

Proof. Let $\mathcal{B}_{v}$ be a sharply transitive bowtie decomposition of $K_{v}$ with respect to $G$. Let $\mathcal{R}=\left\{B_{1}, \ldots, B_{\mu}\right\}$ be a complete system of distinct representatives for the $G$-orbits of $\mathcal{B}_{v}$.

As already remarked, for every $i=1, \ldots, \mu$ we have that $B_{i}$ has trivial stabilizer in $G$, hence $B_{i}^{G}$ has length $v$. Each $B_{i}^{G}$ contains exactly $6 v$ edges of $K_{v}$. Hence $\mu=|E| / 6 v$, that is $v \equiv 1(\bmod 12)$.

We give a result concerning Steiner triple systems. We shall use it in the proof of Proposition 14.

Lemma 5. Assume $v \equiv 1(\bmod 6)$ and let $\mathcal{S}$ be a sharply transitive $S T S(v)$ with respect to a group $G$. Then the number of $G$-orbits of triples of $\mathcal{S}$ is $(v-1) / 6$. 
Proof. Let $L$ denote the stabilizer in $G$ of a triple $T=(x, y, z)$ of $\mathcal{S}$ and assume $h \in L$ with $h \neq i d_{V}$. Since $G$ is sharply transitive on $V$, we have that $h$ fixes no vertex of $T$. Consequently, the representation of $h$ as a product of disjoint cycles contains one of the 3 -cycles $(x y z)$ or $(x z y)$, showing that 3 divides $o(h)$, which in turns divides $|G|=v$. Hence $3 \mid v$, contradicting $v \equiv 1(\bmod 6)$. We conclude $L=\left\langle i d_{V}\right\rangle$, each $G$-orbit of triples of $\mathcal{S}$ has length $v$ and the total number of such orbits is $(v(v-1) / 6) / v=(v-1) / 6$.

Proposition 14. Let $v \equiv 1(\bmod 12)$. The existence of a sharply transitive $S T S(v)$, with respect to a group $G$, is equivalent to the existence of a sharply transitive decomposition $\mathcal{B}_{v}$ with respect to $G$.

Proof. As remarked at the beginning of Section 3, every $\mathcal{B}_{v}$ which is invariant with respect to a group $G$ gives rise to an $\operatorname{STS}(v)$ which is invariant with respect to $G$.

We show that a sharply transitive $\operatorname{STS}(v)$ gives rise to a sharply transitive $B_{v}$. We identify the vertex-set $V$ of $K_{v}$ with the set $\{0,1, \ldots, v-1\}$. Let $\mathcal{S}$ be an $\operatorname{STS}(v)$ on $V$ which is sharply transitive with respect to $G$.

Let $\mathcal{T}$ be a complete system of distinct representatives for the $G$-orbits of $\mathcal{S}$. By Lemma 5 , we have $|\mathcal{T}|=(v-1) / 6$.

Because $G$ is transitive on the vertex-set of $K_{v}$, we can assume that each $T_{i} \in \mathcal{T}$ contains the vertex labeled with 0 , that is we can set $T_{i}=\left(0, x_{i}, y_{i}\right)$, for every $T_{i} \in \mathcal{T}$.

Since $|\mathcal{T}|=(v-1) / 6$ is an even, as $v \equiv 1(\bmod 12)$, and each pair of distinct triples of $\mathcal{T}$ share the vertex 0 , we can pair the elements of $\mathcal{T}$ to form $(v-1) / 12$ distinct bowties with centre 0 . We denote by $\mathcal{R}$ the set of $(v-1) / 12$ distinct bowties of $K_{v}$ which we can construct by pairing the elements of $\mathcal{T}$.

Each $B \in \mathcal{R}$ has $G$-orbit of length $v$, since the two triples constituting it have distinct $G$-orbits of length $v$.

It is easy to see that $\mathcal{B}_{v}=\cup_{B \in \mathcal{R}} B^{G}$ is a sharply transitive bowtie decomposition with respect to $G$.

As already mentioned, for every $v \equiv 1(\bmod 12)$, a large class of sharply transitive bowtie decompositions of $K_{v}$ can be obtained from the abelian STS $(v)$ 's which have been constructed in [19]. In particular, for every admissible value of $v$ there exists a sharply transitive bowtie decomposition of $K_{v}$ with respect to the cyclic group of order $v$.

\section{Primitive bowtie decompositions}

Let $G$ be a primitive permutation group of affine type acting transitively on $v$ vertices, where $v$ is an admissible value. The group $G$ possesses a sharply transitive subgroup $H$ of order $v,[9]$. Therefore, if $\mathcal{B}_{v}$ is a bowtie decomposition which is invariant with respect to $G$, then $\mathcal{B}_{v}$ is sharply transitive with respect to $H$. Hence $v \equiv 1(\bmod 12)$, since Proposition 13 holds.

In this section we will focus our attention on a subclass of primitive permutation groups of affine type. Hence, by the previous remarks, we shall consider the admissible values of $v$ which are congruent to $1(\bmod 12)$. 
Let $p$ be an odd prime and let $q$ be a $p$-primitive divisor of $p^{n}-1, n \geqslant 2$, that is $q \mid\left(p^{n}-1\right)$ but $q \nmid\left(p^{m}-1\right)$, for every $m<n$. Zsigmondy's Lemma, [14, Theorem 6.2], assures the existence of such a divisor except when $p+1$ is a $2-$ power and $n=2$. Hence we shall consider those primes $p$ such that if $n=2$ then $p+1 \neq 2^{i}$, for every positive integer $i$.

Let $\mathbf{V}$ be the $n$-dimensional vector space over $G F(p)$ and let $A$ denote the subgroup of size $q$ of the multiplicative group $G F\left(p^{n}\right)^{*}$.

The group $G$, consisting of all permutations $g: \mathbf{V} \rightarrow \mathbf{V}$ such that $g(\mathbf{x})=a \mathbf{x}+\mathbf{b}$, with $a \in A$ and $\mathbf{b} \in \mathbf{V}$, is a primitive permutation group on $\mathbf{V}$.

The group $G$ acts transitively on $\mathbf{V}$, since it contains the translations group $T=\left\{t_{\mathbf{b}}\right.$ : $\left.t_{\mathbf{b}}(\mathbf{x})=\mathbf{x}+\mathbf{b}, \mathbf{b} \in \mathbf{V}\right\}$. Hence, to prove that $G$ acts primitively on $\mathbf{V}$, it suffices to show that the stabilizer $G_{\mathbf{0}}$ is a maximal subgroup of $G$.

Suppose $G_{0}$ is not a maximal subgroup of $G$, that is there exists a proper subgroup $H$ of $G$ such that $G_{\mathbf{0}}<H$. The subgroup $H$ consists of the permutations $g: \mathbf{V} \rightarrow \mathbf{V}$ such that $g(\mathbf{x})=a \mathbf{x}+\mathbf{b}$, where $a \in A$ and $\mathbf{b}$ belongs to a proper additive subgroup of $G F\left(p^{n}\right)$. Hence $|H|=|A| p^{l}=q p^{l}$ with $l<n$. From [12, II.8.7], it follows that $q \mid\left(p^{l}-1\right)$, a contradiction. Hence $G_{0}$ is a maximal subgroup of $G$ and $G$ is a primitive permutation group on $\mathbf{V}$ of affine type.

The next arguments will be useful to prove Proposition 15. We show that each nondiagonal orbital $\Delta$ of $G$ on $\mathbf{V} \times \mathbf{V}$ has size $|\mathbf{V}| q$.

We note that $q \neq 2$, since $q$ is a $p$-primitive divisor of $p^{n}-1$. Hence $|G|=p^{n} q$ is odd, whence no non-diagonal orbital of $G$ is self-paired.

The map $\phi$ which associates each orbital $\Delta$ of $G$ to the set $\{\mathbf{b}:(\mathbf{0}, \mathbf{b}) \in \Delta\}$ is a bijection between the set of orbitals of $G$ and the set of $G_{\mathbf{0}}$-orbits on $\mathbf{V}$. For $\mathbf{b} \in \mathbf{V}$, $\mathbf{b} \neq \mathbf{0}$, we shall denote by $O_{\mathbf{b}}$ the $G_{\mathbf{0}}$-orbit of $\mathbf{b}$. Note that $O_{\mathbf{b}}=\{a \mathbf{b}: a \in A\}$, since $G_{\mathbf{0}}=\left\{g_{a}: g_{a}(\mathbf{x})=a \mathbf{x}, a \in A\right\}$.

Let $\Delta$ be a non-diagonal orbital of $G$ corresponding to $O_{\mathbf{b}}$. Because $G$ contains the group $T$ of translations on $\mathbf{V}$, it is easy to see that $(\mathbf{u}, \mathbf{w}) \in \Delta$ if and only if $\mathbf{w}-\mathbf{u} \in O_{\mathbf{b}}$. Then $|\Delta|$ is the number of pairs $(\mathbf{u}, \mathbf{w})$ such that $\mathbf{w}-\mathbf{u} \in O_{\mathbf{b}}$.

For every $\mathbf{c} \in O_{\mathbf{b}}$ and for every $\mathbf{w} \in \mathbf{V}$, there exists a unique $\mathbf{u} \in \mathbf{V}$ such that $\mathbf{w}-\mathbf{u}=\mathbf{c}$. In other words, since $G$ is transitive on $\mathbf{V}$, for every $\mathbf{c} \in O_{\mathbf{b}}$ there exist $|\mathbf{V}|$ distinct pairs of distinct elements of $\mathbf{V}$ belonging to $\Delta$. We have $|\Delta|=|\mathbf{V}| \cdot\left|O_{\mathbf{b}}\right|=|\mathbf{V}| q$.

We set $v=|\mathbf{V}|=p^{n}$. Since every non-diagonal orbital of $G$ has size $v q$, we have that the rank $r=\left(|\mathbf{V}|^{2}-|\mathbf{V}|\right) /(|\mathbf{V}| q)+1=(v-1) / q+1$.

Proposition 15. Let $p$ be a prime and let $v=p^{n}, n \geqslant 2$, such that $v \equiv 1(\bmod 12)$ and $p+1$ is not a 2-power if $n=2$. Let $q$ be a p-primitive divisor of $p^{n}-1$. If $(v-1) / q \neq \equiv$ (mod 12), then there is no bowtie decomposition of $K_{v}$ which is invariant with respect to G.

Proof. We identify the vertices of the complete graph with the elements of the vector space $G F\left(p^{n}\right)$.

As already remarked, no non-diagonal orbital of $G$ is self-paired, hence the rank $r$ can be written as $r=2 t+1$ and $t$ corresponds to the number of $G$-orbits of edges. By 
the previous remarks, $r=(v-1) / q+1$, hence $t=(v-1) / 2 q$.

Suppose there exists a bowtie decomposition $\mathcal{B}_{v}$ of $K_{v}$ which is invariant with respect to $G$. Each bowtie of $\mathcal{B}_{v}$ is of type (4), since $|G|$ is odd. A bowtie of type (4) contains exactly 6 edges from distinct edge-orbits. Whence the number of $G$-orbits of edges is divisible by 6 , that is $6 \mid(v-1) / 2 q$, a contradiction.

Using the AllPrimitiveGroups library of GAP, [10], we found primitive permutation groups acting transitively on $v \equiv 1(\bmod 12)$ vertices and of order $v q$, where $v \in\{37,61,73,109,157,181,229\}$ and $q$ is an odd prime divisor of $(v-1)$ such that $(v-1) / q \equiv 0(\bmod 12)$. For each group $G$ we constructed a set of $(v-1) /(12 q)$ distinct representatives of $G$-orbits of bowties yielding a $G$-invariant bowtie decomposition $\mathcal{B}_{v}$. Each $G$-orbit has length $v q$. Unfortunately, we were not able to find a general construction.

\section{1-rotational bowtie decompositions}

Let $K_{v}=(V, E)$ be the complete graph on $v$ vertices. Let $G$ be a subgroup of the symmetric group $\operatorname{Sym}(v)$ which fixes one vertex of $V$ and acts sharply transitively on the remaining vertices, that is $G$ is 1 -rotational on $V$.

Because of the action of $G$ on $V$, we can identify $V$ with $G \cup\{\infty\}$, where $\infty$ is an element not lying in $G$. Observe that $|G|=v-1$.

We will understand the group $G$ in additive form and $0_{G}$ will denote the identity element of $G$. The action of $G$ on the vertices of $K_{v}$ is given by the regular right representation of $G$, that is $g(x)=x+g$ for every $x, g \in G$, with the rule $\infty+g=\infty$.

Proposition 16. Let $v$ be an admissible value. There is no 1-rotational bowtie decomposition of order $v$.

Proof. Suppose there exists a bowtie decomposition $\mathcal{B}_{v}$ which is invariant with respect to $G$.

Let $\left\{B_{1}, \ldots, B_{\mu}\right\}$ be a complete system of distinct representatives for the $G$-orbits of $\mathcal{B}_{v}$. Because of the action of $G$ on the vertices of $K_{v}$, there exists a unique $B_{i} \in$ $\left\{B_{1}, \ldots, B_{\mu}\right\}$ whose $G$-orbit contains all edges which are incident with $\infty$. Observe that the edges which are incident with $\infty$ all lie in the same $G$-orbit, since $G$ acts transitively on $V \backslash\{\infty\}$.

Without loss of generality we can set $B_{i}=B_{1}$ and $B_{1}=\left(x, y_{1}, z_{1}, y_{2}, z_{2}\right)$. Observe that $B_{1}$ contains 4 or 2 edges which are incident with $\infty$, according to whether $x=\infty$ or $x \neq \infty$.

Suppose $x \neq \infty$. Let $S$ be the stabilizer in $G$ of $B_{1}$. Every element of $S$ fixes $x$, since $x$ is the unique 4 -valent vertex of $B_{1}$. Hence $S=\left\langle i d_{V}\right\rangle$, since $x \neq \infty$ and $G$ acts sharply transitively on $V \backslash\{\infty\}$. It follows that $\left|B_{1}^{G}\right|=v-1$, that is $B_{1}^{G}$ contains $2(v-1)$ edges which are incident with $\infty$, a contradiction. Hence $x=\infty$ and $\left|B_{1}^{G}\right|=(v-1) / 4$, as $B_{1}$ contains 4 edges which are incident with $\infty$. 
Each representative in $\left\{B_{2}, \ldots, B_{\mu}\right\}$ has trivial stabilizer in $G$, as its centre lies in $V \backslash\{\infty\}$ and $G$ acts sharply transitively on $V \backslash\{\infty\}$. Hence $\mu=\left|\mathcal{B}_{v} \backslash B_{1}^{G}\right| /(v-1)=$ $[(v(v-1)) / 12-(v-1) / 4] /(v-1)=(v-3) / 12$. That yields a contradiction, since $v \equiv 1,9$ $(\bmod 12)$. Hence there is no bowtie decomposition $\mathcal{B}_{v}$ which is invariant with respect to $G$.

\section{References}

[1] P. Adams, Lambda-fold 2-perfect bowties, Utilitas Math. 44 (1993), 243-253.

[2] J.C. Bermond, C. Huang, A. Rosa, D. Sotteau, Decomposition of complete graphs into isomorphic subgraphs with five vertices, Ars Combin. 10 (1980), 211-254.

[3] E.J. Billington, C.C. Lindner, Maximum packings of bowtie designs, J. Combin. Math. Combin. Comput. 27 (1998), 227-249.

[4] E.J. Billington, C.C. Lindner, The spectrum for 2-perfect bowtie systems, Discrete Math. 135 (1994), 61-68.

[5] M. Buratti, 1-rotational Steiner Triple Systems Over Arbitrary Groups, J. Combin. Des. 9 (2001), 215-226.

[6] C.J. Cho, Rotational Steiner triple systems, Discrete Math. 42 (1982), 153-159.

[7] C.J. Colbourn, A. Rosa, Triple systems, Clarendon Press, Oxford (1999).

[8] J. Doyen, A note on reverse Steiner triple systems, Discrete Math. 1 (1972), 315-319.

[9] J. Dixon, B. Mortimer, Permutation groups. Graduate Texts in Mathematics 163, Springer-Verlag, New York (1996).

[10] The GAP Group, GAP-groups, algorithms, and programming, Version 4.4.10, 2007 (http://www.gap-system.org)

[11] P. Horák, A. Rosa, Decomposing Steiner triple systems into small configurations, Ars Combin. 26 (1988), 91-105.

[12] B. Huppert, Endliche Gruppen I, Springer, Berlin (1967).

[13] Z. Jiang, On rotational Steiner triple systems: five, seven and eleven, Ars Combin. 47 (1997), 201-221.

[14] H. Lüneburg, Translation Planes, Springer, Berlin (1980).

[15] R.A. Mathon, K.T. Phelps, A. Rosa, Small Steiner triple systems and their properties, Ars Combin. 15 (1983), 3-110.

[16] K.T. Phelps, A. Rosa, Steiner triple systems with rotational automorphisms, Discrete Math. 33 (1981), 57-66.

[17] A. Rosa, On reverse Steiner triple systems, Discrete Math. 2 (1972), 61-71.

[18] R.G. Stanton, I.P. Goulden, Graph factorization, general triple systems, and cyclic triple systems, Aequationes Math. 22 (1981), 1-28. 
[19] P. Tannenbaum, Abelian Steiner triple systems, Canad. J. Math. 28 (1976), 12511268.

[20] V.D. Tonchev, Transitive Steiner triple systems of order 25, Discrete Math. 67 (1987), 211-214. 